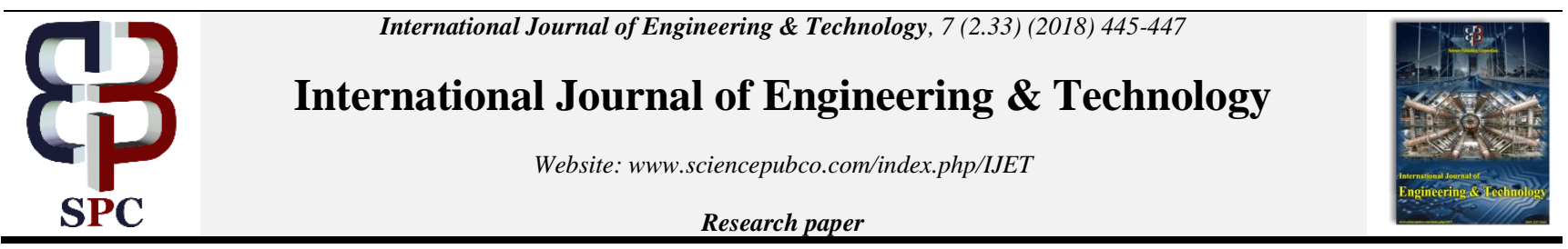

\title{
Analysis for guaranteeing performance in map reduce systems with hadoop and $R$
}

\author{
L. Anand ${ }^{1}$, K. Senthilkumar ${ }^{1}$, N. Arivazhagan ${ }^{1}$, V. Sivakumar ${ }^{1}$ \\ ${ }^{1}$ Assistant Professor, SRM Institute of Science and Technology, Chennai- 603203 \\ *Corresponding author E-mail: anand.l@ktr.srmuniv.ac.in
}

\begin{abstract}
Corporates have fast developing measures of information to technique and store, an information blast goes ahead by USA. By and by one on the whole the chief regular ways to deal with treat these gigantic data amounts region units upheld the MapReduce parallel programming worldview. Though its utilization is across the board inside the exchange, guaranteeing execution limitations, while at a comparable time limiting costs, still gives escalated challenges. We have an angle to have a trend to propose a harsh grained administration hypothetical approach, bolstered procedures that have effectively attempted their quality inside the administration group. We have an angle to have a leaning to acquaint the essential equation with make dynamic models for substantial data MapReduce frameworks, running a matching business. What are a lot of we have a gradient to have a tendency to learn a join of central administration utilize cases: loose execution minor asset and strict execution. For the essential case we have a slant to have a leaning to build up a join of blame administration systems. An established criticism controller and a decent essentially based input that limits the measure of bunch reconfigurations still. In addition, to deal with strict execution necessities a bolster forward ambiguous controller that speedily stifles the ramifications of huge work estimate varieties is created. Every one of the controllers unit substantial on-line all through a benchmark running all through a genuine sixty hub MapReduce bunch, utilizing a data serious Business Intelligence work. Our investigations show the accomplishment of the administration courses used in soothing administration time requirements.
\end{abstract}

Keywords: Mapreduce; Bigdata; Hadoop; Data Processing.

\section{Introduction}

The measure of crude information delivered by everything from our cell phones, tablets, PCs to our brilliant watches is expanding exponentially. Subsequently organizations confront novel and developing difficulties in information stockpiling and investigation. The sheer measure of information accessible is requesting a move of point of view from the customary database ways to deal with stages equipped for taking care of petabytes of unstructured data accessible for undertakings, for example, customized promoting, propelled information mining or arrangement. A standout amongst the most famous of such stages is the MapReduce structure, which is one of the as of now most used programming worldview being used for parallel, circulated calculations over a lot of information. MapReduce is sponsored by the biggest Big Data industry pioneers. For instance, Google has lot of data than 100 thousand MapReduce employments executed day by day , Yahoo has more than 40 thousand PCs running MapReduce occupations, LinkedIn assesses more than 120 billion connections for every day utilizing MapReduce while, Facebook's biggest MapReduce group contains more than a 100 petabytes of information.

\section{Existing system}

Existing thought manages giving backend by abuse MySQL that holds the stack of disadvantages that is learning constraint is that interim is high once (the information the info the data) is enor- mous and once information is lost we tend to can't recoup in this way consequently we tend to proposing thought by abuse Hadoop as well.

\subsection{Drawbacks}

The upkeep of differed records and system of scope ar being done physically by the substance division. This winds up in a few downsides some of that are:

1) It can handle up to GB data.

2) If increase the length of the records performance will be reduced.

3) It can process only structure data

4) Maintaining cost also very high.

\section{Proposed work}

Proposed concept deals with providing database by using hadoop tool we can analyze no limitation of data and simple add number of machines to the cluster and we get results with less time, high throughput and maintenance cost is very less and we are using joins, partitions and bucketing techniques in hadoop.

\subsection{Advantages}

1) Hadoop is more scalable.

2) Hadoop is more economical.

3) It can store UN limited data. 
4) It can process any type of data like structure, UN structure and semi structure.

5) It gets high throughput.

6) It is highly fault-tolerant systems.

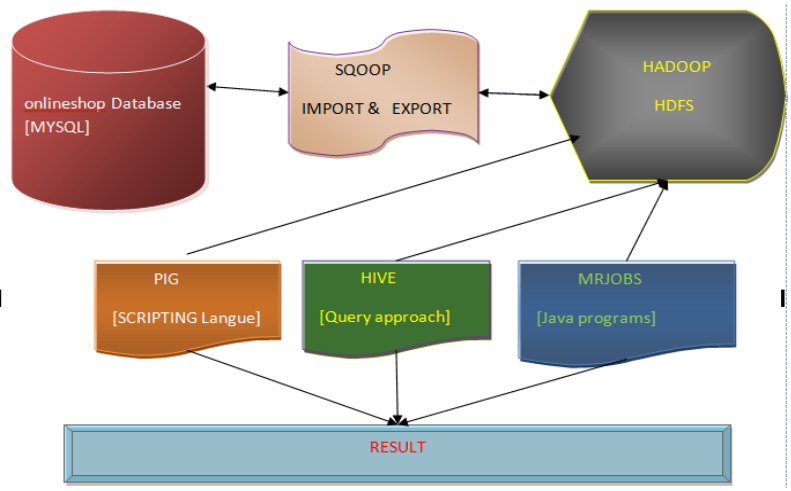

Fig. 1: Architecture of the System. Module Description.

\subsubsection{Data preprocessing module}

In this module we have to create Data set for Feedback dataset it contain set of table such that User details, Baby Item details, Electronic Item details, sports Item details, Men Item Details and feedback or transactions details for last one years .and this data first provide in MySQL database with help of this dataset we analysis this project

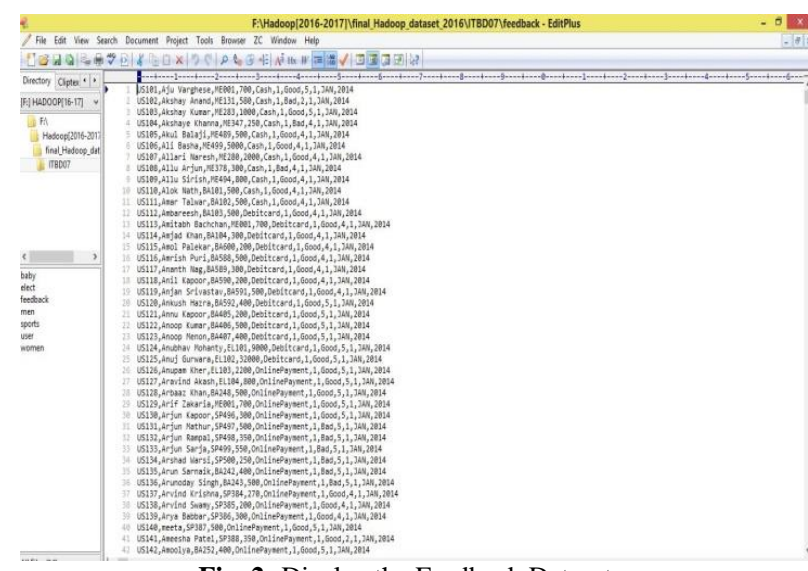

Fig. 2: Display the Feedback Datasets.

\subsubsection{Data ingesion with sqoop}

In This Now we've got a bent to be ready with dataset. so presently our aim is transfer the dataset into Hadoop (HDFS), which can be happen throughout this module. Sqoop might be a commandline interface application for transferring information between relative databases and Hadoop. In this module the dataset is obtained from hadoop (HDFS) mistreatment sqoop Tool. Mistreatment sqoop we have got to perform heap, such if we might prefer to fetch the particular column or if we might prefer to fetch the knowledgeset with specific condition which can be support by Sqoop Tool and knowledge area unit detain hadoop (HDFS).

\subsubsection{Data analytic with hive}

Hive could also be a information ware house system for Hadoop. It runs SQL like queries mentioned as HQL (Hive question language) that gets internally converted to map reduce jobs. Hive was developed by Facebook. Hive supports information definition Language (DDL), information Manipulation Language (DML) and user printed functions. during this module we have got to analysis the dataset practice HIVE tool that is ready to be hold on in hadoop (HDFS).For analysis dataset HIVE practice HQL Language. practice hive we tend to tend to perform Tables creations, joins, Partition, Bucketing plan. Hive analysis the only real Struc- ture Language. Evolutionary algorithm is one of the method to solve this type of problems [11], [12].

\subsubsection{Data analytic with pig}

Apache Pig may be a high level info flow platform for execution Map reduces programs of Hadoop. The language for Pig is pig Latin. Pig handles every structure and unstructured language. It's collectively high of the map reduce methodology running background. In this module collectively used for analyzing the information set through Pig practice Latin Script knowledge flow language.in this collectively we've got an inclination to try to to all operators, functions and joins applying on the data see the result.

\subsubsection{Data analytic with mapreduce}

MapReduce might be a method technique and a program model for distributed computing supported java. The MapReduce rule contains a pair of necessary tasks, specifically Map and reduce. In this module in addition used for analyzing the data set exploitation MAP reduces. Map reduce surpass Java Program.

Map (k1, v1) --->list (K2, v2)

Reduce (K2, list (v2)) ---> list (v3)

function map(String name, String document):

// name: document name

// document: document contents

for each word $w$ in document:

emit $(w, 1)$

function reduce(String word, Iterator partialCounts):

// word: a word

// partialCounts: a list of aggregated partial counts

sum $=0$

for each pc in partialCounts:

sum $+=\mathrm{pc}$

emit (word, sum)

\subsubsection{Data analytic with $R$}

$\mathrm{R}$ is a programming language and software environment for statistical analysis, graphics representation and reporting. $\mathrm{R}$ is a well-developed, simple and effective programming language which includes conditional loops user defined recursive functions and input and output facilities. it has an effective data handling and storage facility.it provides a suite of operators for calculations on arrays, lists, vectors and matrices. The multiple mathematical expression with text is combined with the use of paste () inside expression(), as in the following.
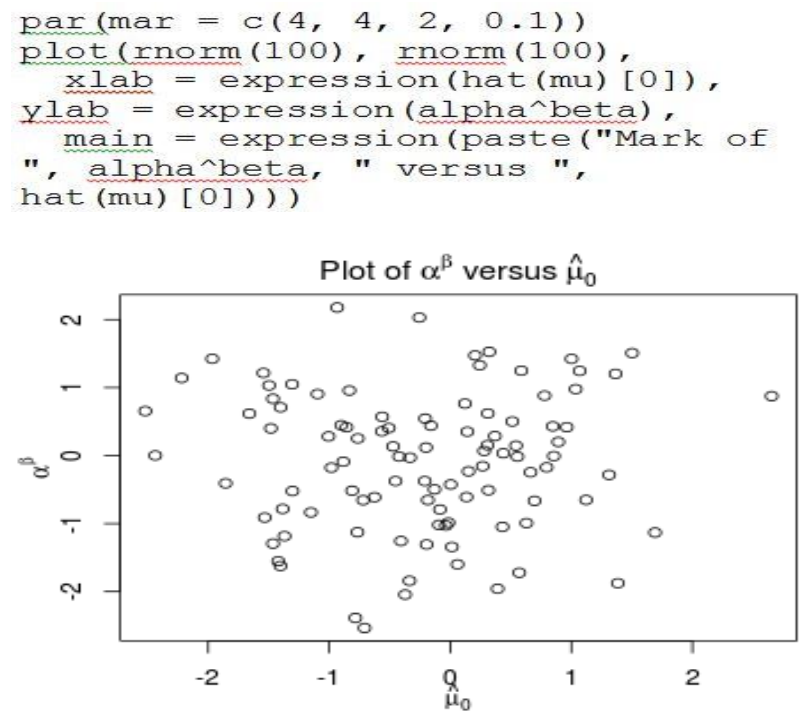
Fig. 3:

Finally, if we want to include variables from an $\mathrm{R}$ session in mathematical expressions, and substitute in their actual values, we can use substitute ().
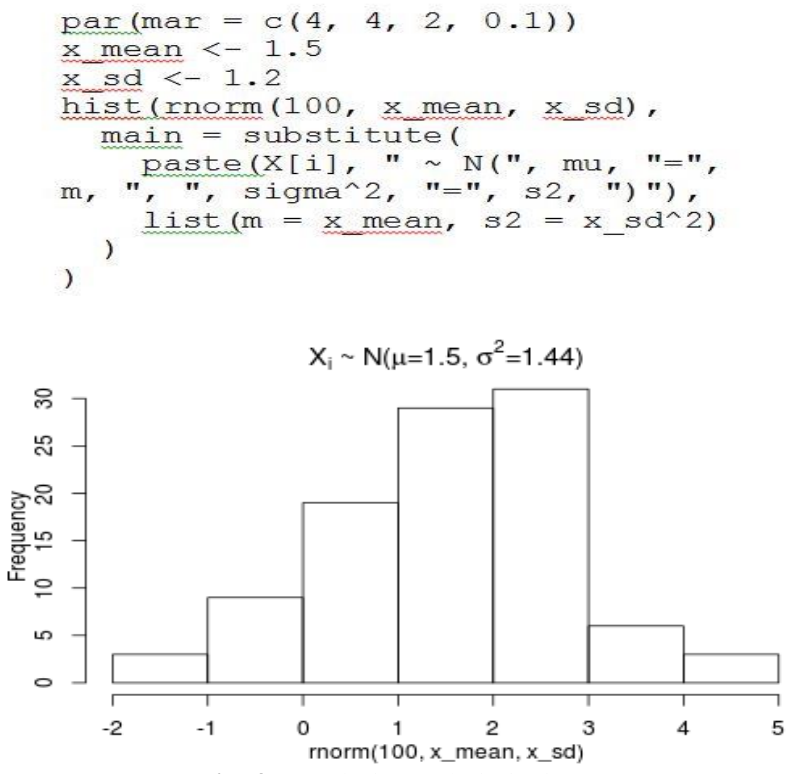

Fig. 4: Conclusion and Limitation.

A method for predicting future merchandise sales in on-line looking has been developed exploitation options extracted from client feedback. The goal of this study was to research that merchandise square measure most liked supported user rating and review, that person highest purchased, that person highest quantity spent and monthly wise conjointly notice all product sales on previous year because the forecast for the subsequent year that kind of merchandise maintains and improve the business. We tend to square measure exploitation spark we will get result hundred times quicker than Hadoop. The key is that it runs in-memory on the cluster, which it is not tied to Hardtop's MapReduce two-stage paradigm. This makes recurrent access to identical information a lot of quicker. Spark will run as a standalone or on high of Hadoop YARN, wherever it will browse information directly from HDFS.

\section{References}

[1] C. Tian, H. Zho, "A module MapReduce scheduler for varied workloads," in Proceedings of the eighth International Conference on Grid and Cooperative Computing (GCC), Washington, DC, Aug. 2008, pp. 217-225.

[2] J. Dean and S. Ghemawat, "MapReduce: simplified evidence handling on huge bunches," Communications of the ACM, vol. 56, no. 1, pp. 108-118, 2008.

[3] Y. Shen, Investing the New Era of Cloud Computing: Data Safety, Transmission, and Executive, 1st ed. Hershey, PA.M, USA: IGI Global, 2014.

[4] M.NB. Zaharia, M. J. Franklin, S. Shenker, and I. Sto- ica, "Spark: bunch registering with working sets," in Proceedings of the second USENIX meeting on Hot subjects in distributed computing, 2010, pp.

[5] J. L. Hellerstein, Y. Diao, S. Parekh, and D. M. Tilbury, Criticism control of processing frameworks. John Wiley and Sons, Inc., New Jersey, 2005.

[6] A. G. Garc'ıa, I. B. Espert, and V. H. Garc'ıa, "Sla-driven element cloud asset administration," Future Generation Computer Systems vol. 31, pp. 1-11, 2014.

[7] E. Casalicchio and L. Silvestri, "Instruments for sla provisioning in cloud-based pro centers," Computer Networks, vol. 57, no. 3, pp. 795-810, 2013.

[8] D.Serrano,S.Bouchenak, Y.Kouki, J. Lejeune, J. Sopena, L. Arantes and P. Sens, 'SLA guarantees for cloud admins," Upcoming Group Computer Systems, no. 0, pp. -, 2015.
[9] S. Durand and N. Marchand, "Also comes to fruition now and again based pid controller," in Proceedings of the European Control Conference (ECC), 2010

[10] S. Rao, R. Ramakrishnan, A. Silberstein, M. Ovsiannikov, and D. Reeves, "Sailfish: Aframeworkforlargescaledataprocessing,"inProceedingsoftheThirdACMSymposiumonCloud Computing, ser. SoCC '12. New York, NY, USA: ACM, 8438103112

[11] Sivakumar, V. \& Rekha, "Node scheduling problem in underwater acoustic sensor network using genetic algorithm" D. Pers Ubiquit Comput (2018). https://doi.org/10.1007/s00779-0181136-3.

[12] Sivakumar V, Rekha D (2018) underwater acoustic sensor node scheduling using an evolutionary memetic algorithm. Res J Telecommun Inf Technol 1:88-94. 